\title{
Factores abióticos sobre aspectos ecofisiológicos de Handroanthus impetiginosus y Handroanthus serratifolius
}

\author{
Abiotic factors on ecophysiological aspects \\ of Handroanthus impetiginosus and Handroanthus serratifolius
}

\begin{abstract}
João Everthon da Silva Ribeiro ${ }^{a^{*},}$ Ester dos Santos Coêlho ${ }^{b}$
"Corresponding author: a State University of Maranhão (UEMA), Department of Technology in Agribusiness Management, Strees Hernani Pereira n²58, Itapecuru-Mirim, Maranhão, Brazil, tel.: +558398171-6327, j.everthon@hotmail.com

${ }^{\mathrm{b}}$ Federal Rural University of Semi-arid (UFERSA), Department of Plant Sciences, Mossoró, Rio Grande do Norte, Brazil.
\end{abstract}

\begin{abstract}
SUMMARY
The objective of this research was to evaluate the influence of abiotic factors on the ecophysiological aspects of Handroanthus impetiginosus and Handroanthus serratifolius throughout the day, as well as to indicate the ideal time for ecophysiological evaluations. The design was entirely randomized, with ten treatments and six repetitions for each species. The treatments used were ten evaluation times throughout the day ( $8 \mathrm{~h}-17 \mathrm{~h}$ ) with an interval of one hour between them. Climatic variables evaluated were internal and external temperature, relative humidity of the internal and external air of the greenhouse and the photosynthetically active radiation of the environment. Ecophysiological aspects evaluated were: net $\mathrm{CO}_{2}$ assimilation, stomatal conductance, transpiration, internal $\mathrm{CO}_{2}$ concentration, vapor pressure deficit, instantaneous water use efficiency, intrinsic water use efficiency and instantaneous carboxylation efficiency. The canonical correlation analysis and principle component analysis were used to verify associations between climatic and ecophysiological variables. In both species, climatic variables were correlated with ecophysiological variables, with higher association between photosynthetically active radiation, internal and external temperature of environment, with the rate of net $\mathrm{CO}_{2}$ assimilation, stomatal conductance, transpiration, instantaneous carboxylation efficiency and vapor pressure deficit. The influence of climatic factors on the ecophysiological aspects of H. impetiginosus and H. serratifolius was verified. In both species, the ideal time of day for measuring ecophysiological variables is between $11 \mathrm{~h}$ and $13 \mathrm{~h}$.
\end{abstract}

Key words: Bignoniaceae, gas exchange, ipê-amarelo, ipê-roxo, irradiance.

\section{RESUMEN}

El objetivo de la investigación fue evaluar la influencia de los factores abióticos en aspectos ecofisiológicos de Handroanthus impetiginosus y Handroanthus serratifolius a lo largo del día, así como indicar el momento ideal para las evaluaciones ecofisiológicas. Se utilizo un diseño experimental completamente aleatorizado, con diez tratamientos y seis repeticiones para cada especie. Los tratamientos utilizados fueron diez momentos de evaluación a lo largo del día ( 8 h-17 h) con un intervalo de una hora entre ellos. Las variables meteorológicas evaluadas fueron la temperatura del aire interior y exterior, y la humedad relativa del aire interior y exterior del ambiente. Los aspectos ecofisiológicos evaluados fueron: tasa de asimilación neta de $\mathrm{CO}_{2}$, conductancia estomática, transpiración, concentración interna de $\mathrm{CO}_{2}$, déficit de presión de vapor, eficiencia instantánea en el uso del agua, eficiencia intrínseca en el uso del agua y eficiencia instantánea de carboxilación. El análisis de la correlación canónica y los componentes principales se utilizaron para verificar las asociaciones entre las variables meteorológicas y ecofisiológicas. En ambas especies, las variables meteorológicas se correlacionaron con las variables ecofisiológicas, con una mayor asociación entre la radiación fotosintéticamente activa, temperatura interna y externa del ambiente, con la tasa de asimilación neta de $\mathrm{CO}_{2}$, conductancia estomática, transpiración, eficiencia instantánea de carboxilación y déficit de presión de vapor. Se verificó la influencia de los factores meteorológicos en los aspectos ecofisiológicos de H. impetiginosus y H. serratifolius. En ambas especies, la hora ideal del día para medir las variables ecofisiológicas es entre las $11 \mathrm{~h} \mathrm{y} \mathrm{las} 13 \mathrm{~h}$.

Palabras clave: Bignoniaceae, intercambio gaseoso, ipê-amarelo, ipê-roxo, irradiación.

\section{INTRODUCCIÓN}

Conocido popularmente como ipê-roxo, pau d'arco roxo y ipê-roxo-da-mata, Handroanthus impetiginosus (Mart. ex DC.) Mattos (Bignoniaceae Juss.) es una especie de árbol caducifolio nativo de Brasil, que puede alcanzar hasta $20 \mathrm{~m}$ de altura. Tiene una amplia distribución geográfica, desde México hasta el norte de Argentina, y en Brasil se da en las regiones Norte, Noreste, Centro-Oeste y Sureste. Se encuentra en todos los dominios fitogeográficos, ex- 
cepto en la Pampa, en altitudes que varían desde el nivel del mar hasta los 1.400 metros. Esta especie presenta una gran importancia económica, ya que se utiliza principalmente para fines madereros en la construcción de muebles, además, la corteza tiene compuestos químicos bioactivos que se utilizan con fines medicinales con potencial para actividades antiinflamatorias, antibióticas y analgésicas (Beltreschi et al. 2019). Se utiliza en el paisajismo en general y en la recuperación de zonas degradadas (Ferreira et al. 2020), como también tiene un potencial alimenticio para la fauna, por ejemplo, las abejas (Benevides y Carvalho 2009).

Handroanthus serratifolius (Vahl) S. Grose, conocido popularmente como ipê-amarelo y pau d'arco amarelo, es una especie de árbol de tamaño medio a grande, con una altura que varía entre 5 y $25 \mathrm{~m}$. Se distribuye en Colombia, el Ecuador, Guyana, Bolivia, la Guayana Francesa, el Perú, Suriname, Venezuela y el Brasil. En el Brasil está ampliamente distribuida y se da en varios dominios fitogeográficos, que van desde el Amazonas hasta la región meridional del país. La madera de la especie tiene importancia económica principalmente en la construcción civil y con fines tecnológicos, como los mangos de herramientas y los muebles (Oliveira et al. 2019). La corteza del tronco tiene propiedades farmacológicas, utilizándose en la medicina tradicional para el tratamiento de inflamaciones generales, enfermedades respiratorias, así como metabolitos secundarios con actividades antipalúdicas, antitumorales y antiparasitarias (Costa et al. 2017). Se cultiva ampliamente en las zonas urbanas para la ornamentación y puede utilizarse en la recuperación de zonas degradadas y en la reforestación, adaptándose a los suelos secos de baja fertilidad (Vieira y Weber 2017).

Debido a la importancia de estas especies para las regiones de ocurrencia, se necesitan estudios para evaluar el comportamiento y el desempeño ecofisiológico debido a los diferentes factores abióticos. Entre los principales factores que influyen en la fisiología de las plantas, destacan la radiación solar (irradiación), la temperatura del aire y la humedad relativa (Lambers y Oliveira 2019). Las especies forestales, debido a sus características intrínsecas, pueden presentar respuestas diferentes a las condiciones ambientales y, por lo tanto, la actividad fotosintética de las plantas se ven significativamente alteradas (Dias et al. 2017).

La radiación solar (irradiación) a lo largo del día promueve efectos directos en la fotosíntesis de las plantas para influir en la absorción y transferencia de energía en el aparato fotosintético, así como para causar cambios en la asimilación neta de $\mathrm{CO}_{2}$, la conductancia estomática y la transpiración (Cruces et al. 2017). La temperatura del aire puede influir en el proceso fotosintético de las plantas directa o indirectamente. El efecto indirecto está relacionado con la actividad de los estomas, tales como la apertura y el cierre de los mismos, provocando la regulación de las relaciones hídricas en las hojas a través de la transpiración. El efecto directo de la temperatura del aire sobre la asimilación del $\mathrm{CO}_{2}$ implica la regeneración de Ribulosa 1,5-bi- fosfato (RuBP), así como modificaciones en la actividad de Rubisco (Bhatla y Lal 2018).

En vista de lo anterior, los estudios relacionados con los aspectos ecofisiológicos en las especies forestales, como H. impetiginosus y H. serratifolius, son de gran importancia, por lo que se trata de comprender la adaptación y estabilización de estas especies en condiciones ambientales adversas. Sin embargo, la información en la literatura sobre los mecanismos fisiológicos de estas especies en respuesta a condiciones ambientales adversas es escasa. Esta información puede ser útil en la elaboración de modelos que tengan como objetivo predecir escenarios sobre la fijación de carbono, y también puede ayudar a explicar la dinámica de estas especies en fragmentos de vegetación. La hipótesis de investigación fue que $H$. impetiginosus y $H$. serratifolius presentan diferentes respuestas ecofisiológicas a lo largo del día, con menor rendimiento fotosintético en el período de menor radiación fotosintética (irradiancia) y baja temperatura del aire. Así pues, el objetivo de la investigación fue evaluar la influencia de los factores abióticos en los aspectos ecofisiológicos de $\mathrm{H}$. impetiginosus y H. serratifolius a lo largo del día, así como indicar el momento ideal para las evaluaciones ecofisiológicas.

\section{MÉTODOS}

La investigación se llevó a cabo en un invernadero, perteneciente al Departamento de Ciencias Vegetales y Ambientales de la Universidad Federal de Paraíba (Campus II) (6 $6^{\circ} 57^{\prime} 59^{\prime \prime} \mathrm{S}$ y $\left.35^{\circ} 42^{\prime} 57^{\prime \prime} \mathrm{O}\right)$, ubicado en el municipio de Areia, estado de Paraíba, Noreste de Brasil. La región está situada en la microrregión de Brejo y la mesorregión de Agreste Paraibano y tiene una altitud que varía entre 400 y $600 \mathrm{~m}$, con una temperatura media del aire de $22{ }^{\circ} \mathrm{C}$ y una precipitación anual de $1.400 \mathrm{~mm}$ (Ribeiro et al. 2018). El clima de la región es tropical, caracterizado por ser cálido y húmedo con lluvias de otoño-invierno, clasificado según Alvares et al. (2013) como As. Durante el período experimental, el medio ambiente presentó una temperatura media y una humedad relativa del aire de $24,2^{\circ} \mathrm{C}$ y $53,5 \%$, respectivamente, cuyas mediciones se realizaron con un termohigrómetro digital portátil (Minipa, modelo MT-241A).

Las semillas de $H$. impetiginosus y $H$. serratifolius se recogieron en enero de 2019 en matrices adultas en los remanentes forestales de Caatinga, situados en el municipio de Solânea, ubicado en la mesoregión de Agreste, microrregión de Curimataú Oriental, en el estado de Paraíba, al Noreste de Brasil [altitud de $626 \mathrm{~m}\left(06^{\circ} 46^{\prime} 40^{\prime \prime} \mathrm{S}\right.$ y $\left.\left.35^{\circ} 41^{\prime} 49^{\prime \prime} \mathrm{W}\right)\right]$. Para la producción de plántulas, se recogieron las semillas que alcanzaron la fase de maduración, es decir, con dehiscencia en una etapa temprana. Después de la cosecha, las semillas se seleccionaron manualmente y se sumergieron en una solución de hipoclorito de sodio al $2 \%$ durante cinco minutos para su desinfección antes de la siembra. 
Para la siembra se utilizaron macetas de plástico con capacidad para $8 \mathrm{dm}^{3}$ y un sustrato compuesto de tierra vegetal y Bioplant ${ }^{\mathbb{B}}(3: 1)$, con los atributos químicos presentados en el cuadro 1 . Se utilizaron seis semillas por maceta y a los 40 días de su aparición se realizó un raleo, manteniendo la uniformidad de los individuos de la especie. Durante el período de investigación, la irrigación de las plantas se mantuvo al $80 \%$ de la capacidad de la maceta, siendo controlada mediante lisimetría de drenaje.

Se utilizó un diseño experimental completamente aleatorizado, con diez tratamientos y seis repeticiones para cada especie, dos plantas por unidad experimental (parcela), totalizando 24 individuos. Los tratamientos utilizados fueron diez momentos de evaluación a lo largo del día (8:00 a 17:00 h) con un intervalo de una hora entre ellos.

Las variables meteorológicas evaluadas fueron la temperatura del aire interna (Tin) y externa $($ Tex $)\left({ }^{\circ} \mathrm{C}\right)$, y la humedad relativa interna (HRin) y externa (HRex) del ambiente, con la ayuda de un termómetro digital portátil (Minipa, modelo MT-241A). Para la cuantificación de la radiación fotosintética activa (RFA) $\left(\mu \mathrm{mol} \mathrm{m} \mathrm{m}^{-2} \mathrm{~s}^{-1}\right)$, se utilizó el sensor de luz acoplado al analizador portátil de gas por infrarrojo (IRGA) (Licor, modelo LI-6400XT).

Los aspectos ecofisiológicos evaluados fueron la asimilación neta de $\mathrm{CO}_{2}$ (A) $\left(\mu \mathrm{mol} \mathrm{m} \mathrm{m}^{-2} \mathrm{~s}^{-1}\right)$, conductancia estomacal (gs) $\left(\mathrm{mol} \mathrm{m}^{-2} \mathrm{~s}^{-1}\right)$, transpiración (E) (mmol de $\left.\mathrm{H}_{2} \mathrm{O} \mathrm{m}^{-2} \mathrm{~s}^{-1}\right)$, concentración interna de $\mathrm{CO}_{2}(\mathrm{Ci})(\mu \mathrm{mol}$ de $\mathrm{CO}_{2}$ mol- $^{-1}$ ) y déficit de presión de vapor (DPV). A partir de estas variables se calcularon la eficiencia instantánea del uso del agua (EUA: A/E) $\left[\left(\mu \mathrm{mol} \mathrm{m}^{-2} \mathrm{~s}^{-1}\right) /\left(\mathrm{mmol} \mathrm{H}_{2} \mathrm{O}\right.\right.$ $\left.\mathrm{m}^{-2} \mathrm{~s}^{-1}\right)$ ], eficiencia intrínseca del uso del agua (EiUA: A/ gs) $\left[\left(\mu \mathrm{mol} \mathrm{m} \mathrm{m}^{-2} \mathrm{~s}^{-1}\right) /\left(\mathrm{mol} \mathrm{m}^{-2} \mathrm{~s}^{-1}\right)\right]$ y eficiencia instantánea de carboxilación (EiC: $\mathrm{A} / \mathrm{Ci})\left[\left(\mu \mathrm{mol} \mathrm{m}^{-2} \mathrm{~s}^{-1}\right) /(\mu \mathrm{mol} \mathrm{CO}\right.$ $\left.\mathrm{mol}^{-1}\right)$ ]. Las mediciones se realizaron de acuerdo con el siguiente protocolo: humedad relativa entre $50-60 \%$; flujo de aire de $300 \mu \mathrm{mol} \mathrm{s}^{-1}$, concentración de $\mathrm{CO}_{2}$ de $400 \mu \mathrm{mol}$ mol- $^{-1}$ (mezclador, modelo 6400-01) y sensor de luz natural acoplado a una cámara de hojas de $6 \mathrm{~cm}^{2}$. Para las evaluaciones se utilizó el IRGA, que se realizó en cuatro hojas sanas y completamente expandidas, en el tercio medio de cada individuo.

Las evaluaciones se llevaron a cabo 360 días después de la emergencia, en condiciones ideales de luminosidad (pleno sol), a fin de inferir los efectos de los parámetros meteorológicos en los aspectos ecofisiológicos de los individuos.
Se utilizó el análisis de correlación canónica (ACC) y el análisis de componentes principales (ACP) para verificar las asociaciones entre las variables meteorológicas (RFA, Tin, Tex, HRin y HRex) y las variables ecofisiológicas (A, gs, E, Ci, DPV, EUA, EiUA y EiC), así como la prueba de significación multivariante de Lambda de Wilks (aproximación de la distribución de F) para observar la significación de las raíces canónicas juntas. Los análisis estadísticos se realizaron con el software SAS ${ }^{\circledR}$ 9.4M6.

\section{RESULTADOS}

Según la prueba de significación de Lambda de Wilks, las variables ecofisiológicas presentaron correlaciones con las variables meteorológicas, obteniendo significación en las cuatro primeras parejas canónicas de cada especie estudiada, con valores de $\mathrm{R}^{2}$ que oscilan entre 0,64 y 0,99 en H. impetiginosus, y entre 0,56 y 0,99 en $H$. serratifolius, respectivamente (cuadro 2).

En ambas especies se observó en el primer par canónico que las variables meteorológicas más importantes fueron la radiación fotosintéticamente activa ( $\mathrm{cc}=0,87 \mathrm{y}$ $0,76$ ), la temperatura interna ( $c c=0,75$ y 0,69$)$ y la temperatura externa del ambiente ( $\mathrm{cc}=0,72$ y 0,67$)$, correlacionándose positivamente con la asimilación neta de $\mathrm{CO}_{2}(\mathrm{cc}$ $=0,90$ y 0,83$)$, conductancia estomática $(c c=0,83$ y 0,69$)$, transpiración ( $\mathrm{cc}=0,79$ y 0,77$)$, eficiencia instantánea de carboxilación ( $\mathrm{cc}=0,71$ y 0,79$)$ y déficit de presión de vapor $(\mathrm{cc}=0,66$ y 0,64$)$ (cuadro 3$)$.

En cuanto al análisis de los componentes principales (ACP), se observó que en $H$. impetiginosus los ejes concentraban el 77,54 \% de la variabilidad total de los datos (49,38 \% en CP1 y 28,16\% en CP2), observándose que la asimilación neta de $\mathrm{CO}_{2}$, conductancia estomática, transpiración y eficiencia instantánea de carboxilación estuvieron fuertemente correlacionadas con la radiación fotosintéticamente activa, así como el déficit de la presión de vapor correlacionó con la temperatura interna y la temperatura externa del ambiente (figura 1A). En H. serratifolius las dimensiones del eje $(44,74 \%$ en CP1 y $24,00 \%$ en CP2), concentraron el 68,74\% de la variabilidad total de los datos (figura 1B). Hubo correlaciones positivas entre la radiación fotosintéticamente activa con la asimilación neta de $\mathrm{CO}_{2}$, la transpiración, la eficiencia instantánea de carboxilación y la conductancia estomática, y entre la tem-

Cuadro 1. Análisis de fertilidad del sustrato utilizado en el experimento.

Fertility analysis of the substrate used in the experiment.

\begin{tabular}{|c|c|c|c|c|c|c|c|c|c|c|c|}
\hline \multirow{2}{*}{$\mathrm{pH}\left(\mathrm{H}_{2} 0\right)$} & $\mathrm{P}$ & K & $\mathrm{Na}$ & $\mathrm{H}+\mathrm{Al}$ & $\mathrm{Al}$ & $\mathrm{Ca}$ & $\mathrm{Mg}$ & $\mathrm{SB}$ & CIC & \multirow{2}{*}{$\frac{\mathrm{V}}{\%}$} & \multirow{2}{*}{$\frac{\text { M.O. }}{\mathrm{g} \mathrm{kg}^{-1}}$} \\
\hline & \multicolumn{2}{|c|}{$\mathrm{mg} \mathrm{dm}^{-3}$} & \multicolumn{7}{|c|}{ cmolc dm ${ }^{-3}$} & & \\
\hline 5,42 & 118,7 & 217,2 & 0,43 & 4,62 & 0,00 & 3,50 & 3,10 & 7,58 & 12,2 & 62,1 & 29,86 \\
\hline
\end{tabular}

SB: suma de bases; CIC: capacidad de intercambio catiónico; V: saturación de bases; M.O.: materia orgánica. 
Cuadro 2. Prueba multivariante Lambda de Wilks (aproximación de la distribución F). $\mathrm{R}^{2}$ : correlación canónica; Fa: valor aproximado de F; GL-Num: grado de libertad del numerador; GL-Den: grado de libertad del denominador.

Wilks's Lambda multivariate test (F distribution approximation).

\begin{tabular}{lccccc}
\hline \multicolumn{5}{c}{ Handroanthus impetiginosus } \\
\hline Función canónica & $\mathrm{R}^{2}$ & $\mathrm{Fa}$ & GL-Num & GL-Den & $\operatorname{Pr}>\mathrm{F}$ \\
\hline 1 & 0,99 & 23,64 & 40 & 207,66 & $<0,0001$ \\
2 & 0,91 & 9,21 & 28 & 174,49 & $<0,0001$ \\
3 & 0,76 & 5,40 & 18 & 139,08 & $<0,0001$ \\
4 & 0,64 & 3,78 & 10 & 100,00 & 0,0002 \\
5 & 0,32 & 1,54 & 4 & 51,00 & 0,2037 \\
\hline & \multicolumn{5}{c}{ Handroanthus serratifolius } \\
\hline Función canónica & $\mathrm{R}^{2}$ & Fa & GL-Num & GL-Den & $\operatorname{Pr}>\mathrm{F}$ \\
\hline 1 & 0,99 & 17,21 & 40 & 207,66 & $<0,0001$ \\
2 & 0,82 & 5,28 & 28 & 174,49 & $<0,0001$ \\
3 & 0,62 & 3,46 & 18 & 139,08 & $<0,0001$ \\
4 & 0,56 & 3,16 & 10 & 100,00 & 0,0015 \\
\hline 5 & 0,38 & 2,27 & 4 & 51,00 & 0,0747 \\
\hline
\end{tabular}

Cuadro 3. Correlaciones canónicas y par canónico entre las variables meteorológicas y ecofisiológicas de Handroanthus impetiginosus y Handroanthus serratifolius. ** Significativo al $1 \%$ de probabilidad por prueba de ji-cuadrado; S1: Handroanthus impetiginosus; S2: Handroanthus serratifolius; $\mathrm{R}^{2}=$ correlación canónica.

Canonic correlations and canonical pair between the climatic and ecophysiological variables of Handroanthus impetiginosus and Handroanthus serratifolius.

\begin{tabular}{lcc}
\hline \multicolumn{1}{c}{ Variables } & \multicolumn{2}{c}{ Par canónico } \\
\hline \multicolumn{1}{c}{ Grupo I: Meteorológicas } & $\mathrm{S} 1$ & $\mathrm{~S} 2$ \\
\hline Radiación fotosintética activa (RFA) & 0,87 & 0,76 \\
Temperatura interna (Tin) & 0,75 & 0,69 \\
Temperatura externa (Tex) & 0,72 & 0,67 \\
Humedad relativa- interna (HRin) & $-0,50$ & $-0,39$ \\
Humedad relativa- externa (HRex) & $-0,54$ & $-0,42$ \\
\hline \multicolumn{1}{c}{ Grupo II: Ecofisiológicas } & & \\
\hline Asimilación neta de CO ${ }_{2}$ (A) & 0,90 & 0,83 \\
Conductancia estomacal (g ${ }_{\mathrm{s}}$ ) & 0,83 & 0,69 \\
Transpiración (E) & 0,79 & 0,77 \\
Concentración interna de CO ${ }_{2}(\mathrm{Ci})$ & $-0,49$ & $-0,55$ \\
Déficit de presión de vapor (DPV) & 0,66 & 0,64 \\
Eficiencia instantánea del uso del agua (EUA) & $-0,55$ & $-0,44$ \\
Eficiencia intrínseca del uso del agua (EiUA) & $-0,58$ & $-0,47$ \\
Eficiencia instantánea de carboxilación (EiC) & 0,71 & 0,79 \\
$\mathrm{R}^{2}$ & 0,99 & 0,99 \\
Significación & $* *$ & $* *$ \\
\hline & & \\
\hline
\end{tabular}

peratura del ambiente externo e interno con el déficit de presión de vapor (figura 1B). En ambas especies se observó que los autovectores de las variables ecofisiológicas, excepto la concentración interna de $\mathrm{CO}_{2}$, se localizan en la porción más extrema de la derecha, presentando valores positivos, mientras que la humedad relativa interna y externa se localizaron en la porción externa de la izquierda (con valores negativos), evidenciando la distinción de estos parámetros y los demás analizados (figura 1A y B).

Las variables meteorológicas presentaron variaciones, en las que la radiación fotosintéticamente activa (RFA) aumentó significativamente a lo largo del día, con valores que oscilaron entre $29,01 \mu \mathrm{mol} \mathrm{s}^{-1} \mathrm{~m}^{-2}$ y $1506,14 \mu \mathrm{mol} \mathrm{s}^{-1} \mathrm{~m}^{-2}$, registrados a las $17 \mathrm{~h} \mathrm{y} 12 \mathrm{~h}$, respectivamente (figura 2). La temperatura del ambiente interno y externo alcanzó los valores máximos en el período de $12 \mathrm{~h}$ y $13 \mathrm{~h}$, con valores de $40,6{ }^{\circ} \mathrm{C}$ y $39,9^{\circ} \mathrm{C}$ en el interior de la casa de la vegetación, y $38,5^{\circ} \mathrm{C}$ y $36,7{ }^{\circ} \mathrm{C}$ en el área externa (figura 2). La humedad relativa del aire interior y exterior presentó reducción durante el período de mayor temperatura y radiación fotosintéticamente activa, con los valores más bajos observados a la $13 \mathrm{~h}$ (HRin de $29 \%$ y HRex de $32 \%$ ) y los valores más altos durante el período de $8 \mathrm{~h}$ y $17 \mathrm{~h}$ (figura 2).

La asimilación neta de $\mathrm{CO}_{2}$ (A) mostró un aumento significativo en las primeras horas del día (figura 3A), junto con la radiación fotosintéticamente activa (RFA) y la temperatura interna y externa del ambiente (Tin y Tex). En H. impetiginosus se observaron las mayores tasas de asimilación neta de $\mathrm{CO}_{2}$, con valores registrados a las $11 \mathrm{~h} \mathrm{y} 12 \mathrm{~h}(6,7301 \mu \mathrm{mol}$ $\mathrm{m}^{-2} \mathrm{~s}^{-1}$ y $8,2402 \mu \mathrm{mol} \mathrm{m} \mathrm{m}^{-2} \mathrm{~s}^{-1}$, respectivamente) (figura $3 \mathrm{~A}$ ), 
mientras que en $H$. serratifolius los valores aumentaron hasta las $12 \mathrm{~h} \mathrm{y} 13 \mathrm{~h}\left(5,8567 \mu \mathrm{mol} \mathrm{m} \mathrm{m}^{-2} \mathrm{~s}^{-1}\right.$ y $6,0748 \mu \mathrm{mol}$ $\mathrm{m}^{-2} \mathrm{~s}^{-1}$, respectivamente) (figura $3 \mathrm{~A}$ ). En ambas especies, los valores más bajos se registraron a las 17 h (figura 3A).

La conductancia estomática (gs) mostró un comportamiento similar a la tasa de asimilación neta de $\mathrm{CO}_{2}$, con incrementos graduales a lo largo del día en ambas espe- cies, registrándose en $H$. impetiginosus $0,1505 \mathrm{~mol} \mathrm{~m}^{-2} \mathrm{~s}^{-1}$ y $0,1844 \mathrm{~mol} \mathrm{~m}^{-2} \mathrm{~s}^{-1}$ a las $11 \mathrm{~h} \mathrm{y} 12 \mathrm{~h}, \mathrm{y} 0,1295 \mathrm{~mol} \mathrm{~m}^{-2}$ $\mathrm{s}^{-1}$ y $0,1540 \mathrm{~mol} \mathrm{~m}^{-2} \mathrm{~s}^{-1}$ a las $12 \mathrm{~h}$ y $13 \mathrm{~h}$ en plantas de $H$. serratifolius, con considerables descensos hasta las $17 \mathrm{~h}$ (figura 3B).

La transpiración (E) mostró la misma tendencia que la asimilación neta de $\mathrm{CO}_{2}$ y la conductancia estomática, con
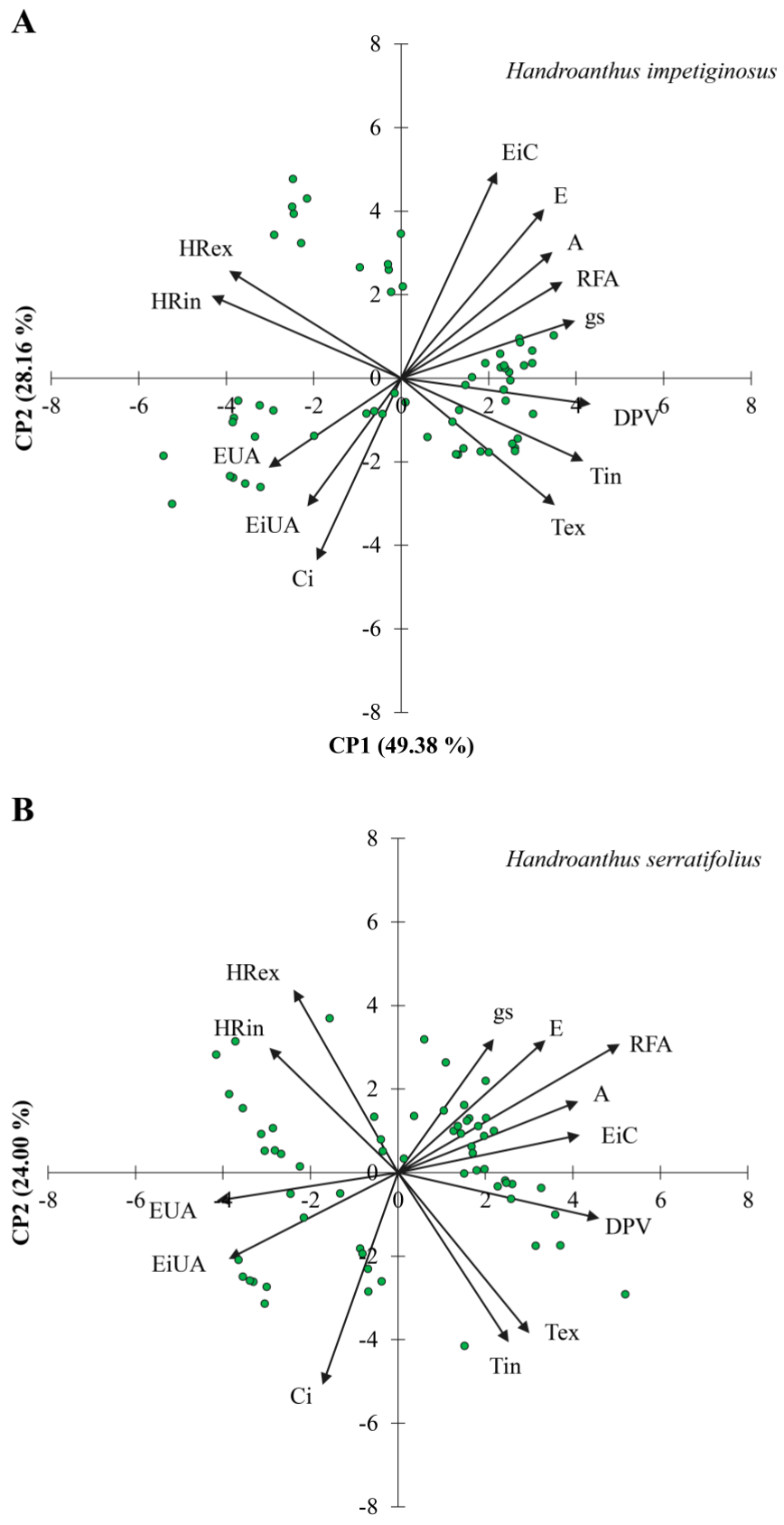

CP1 (44.74\%)

Figura 1. Análisis de los componentes principales (CP1 y CP2) entre las variables meteorológicas y ecofisiológicas de Handroanthus impetiginosus (A) y Handroanthus serratifolius (B). serratifolius (B).

Principal component analysis among the climatic and ecophysiological variables of Handroanthus impetiginosus (A) and Handroanthus 


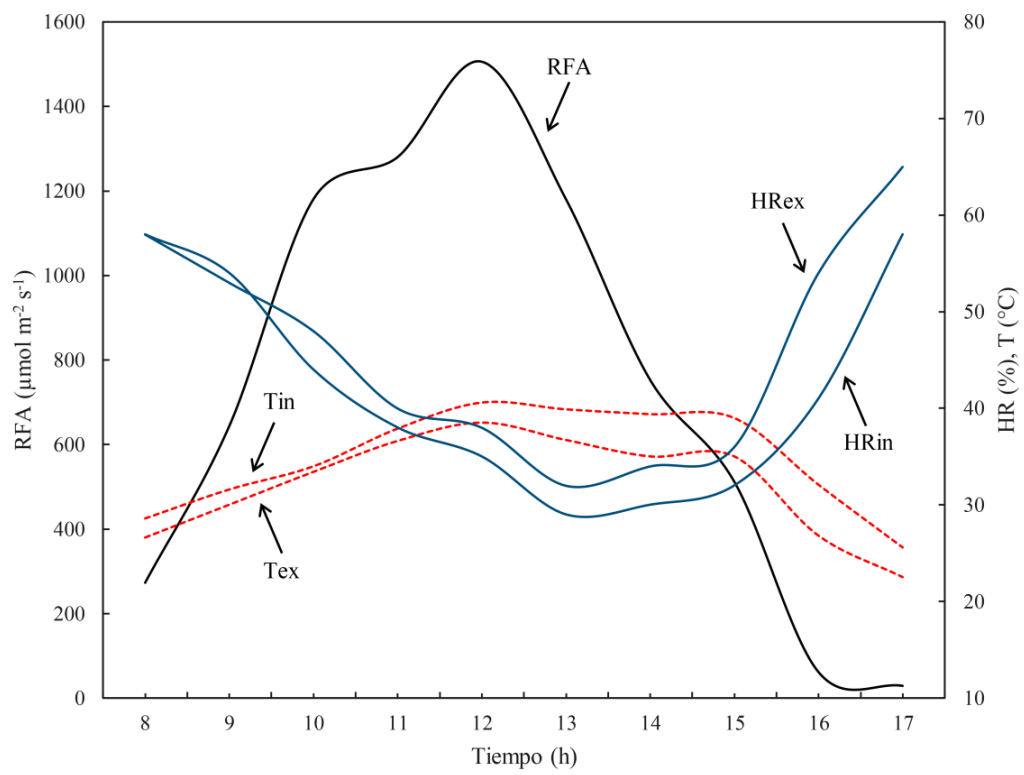

Figura 2. Radiación fotosintéticamente activa (RFA), temperatura interna (Tin) y externa (Tex), y humedad relativa interna (HRin) y externa (HRex) en el invernadero durante la realización del experimento.

Photosynthetically active radiation (RFA), internal (Tin) and external (Tex) temperature, and internal (HRin) and external (HRex) relative humidity in greenhouse during the conduction of the experiment.

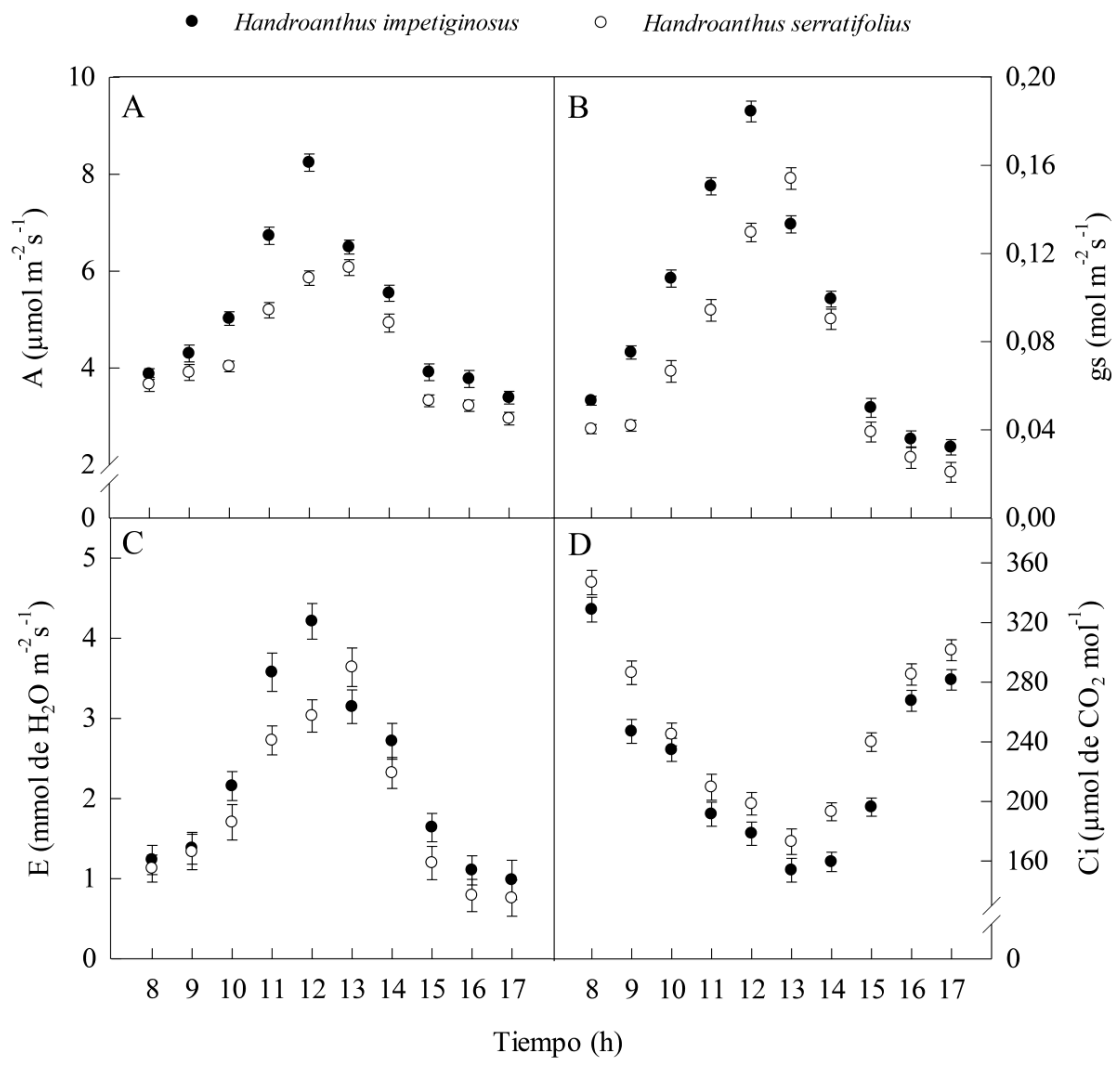

Figura 3. Asimilación neta de $\mathrm{CO}_{2}(\mathrm{~A})(\mathrm{A})$, conductancia estomática (gs) (B), transpiración (E) (C) y concentración interna de $\mathrm{CO}_{2}$ (Ci) (D) en plantas de Handroanthus impetiginosus (•) y Handroanthus serratifolius (O) en función de la hora (h) del día.

Net $\mathrm{CO}_{2}$ assimilation (A) (A), stomatal conductance (gs) (B), transpiration (E) (C) and internal $\mathrm{CO}_{2}$ concentration (Ci) (D) in Handroanthus impetiginosus $(\bullet)$ and Handroanthus serratifolius $(\mathrm{O})$ plants as a function of time $(\mathrm{h})$ of day. 
los valores más altos observados a las $11 \mathrm{~h}$ y $12 \mathrm{~h}$ en $\mathrm{H}$. im-

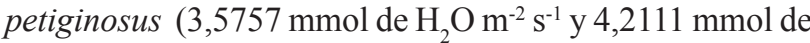
$\mathrm{H}_{2} \mathrm{O} \mathrm{m}^{-2} \mathrm{~s}^{-1}$, respectivamente), y en el período de $12 \mathrm{~h}$ y $13 \mathrm{~h}$ en $H$. serratifoliius $\left(3,0306 \mathrm{mmol} \mathrm{de} \mathrm{H}_{2} \mathrm{O} \mathrm{m}^{-2} \mathrm{~s}^{-1}\right.$ y 3,6384 mmol de $\mathrm{H}_{2} \mathrm{O} \mathrm{m}^{-2} \mathrm{~s}^{-1}$, respectivamente), siendo el más bajo observado a las $17 \mathrm{~h}$ en ambas especies (figura $3 \mathrm{C}$ ).

En cuanto a la concentración interna de $\mathrm{CO}_{2}(\mathrm{Ci})$, se observó un comportamiento inverso en relación con las demás variables, registrándose los valores más altos a las $8 \mathrm{~h}$ y a las $17 \mathrm{~h}$ en las especies estudiadas $(328,69$ y 281,55 $\mu \mathrm{mol}$ de $\mathrm{CO}_{2}$ mol $^{-1}$ en $H$. impetiginosus y 346,83 y 281,55 $\mu \mathrm{mol}$ de $\mathrm{CO}_{2} \mathrm{~mol}^{-1}$ en $H$. serratifolius, respectivamente) (figura 3D).

El déficit de presión de vapor (DPV) aumentó gradualmente a lo largo del día, con valores que oscilan entre 1,54 $\mathrm{kPa}(8 \mathrm{~h})$ y $3,21 \mathrm{kPa}(12 \mathrm{~h})$ en $H$. impetiginosus, y entre 1,34 $\mathrm{kPa}(8 \mathrm{~h})$ y $2,84 \mathrm{kPa}(12 \mathrm{~h}$ ) en $H$. serratifolius (figura $4 \mathrm{~A}$ ).

La eficiencia instantánea del uso del agua (EUA), mostró una reducción durante el período entre las $8 \mathrm{~h}$ y las 13 $\mathrm{h}$ en ambas especies, con una disminución del $34,3 \%$ en H. impetiginosus y del $48,9 \%$ en $H$. serratifolius, entre los valores más altos y los más bajos registrados en este período (figura 4B). Los valores más altos se observaron a las $16 \mathrm{~h}$ en $H$. serratifolius y a las $17 \mathrm{~h}$ en $H$. impetiginosus , con 4,0850 $\left[\left(\mu \mathrm{mol} \mathrm{m}{ }^{-2} \mathrm{~s}^{-1}\right)\left(\mathrm{mmol} \mathrm{de} \mathrm{H}_{2} \mathrm{O} \mathrm{m}^{-2} \mathrm{~s}^{-1}\right)^{-1}\right] \mathrm{y}$ $3,4503\left[\left(\mu \mathrm{mol} \mathrm{m} \mathrm{s}^{-1}\right)\left(\mathrm{mmol} \text { de } \mathrm{H}_{2} \mathrm{O} \mathrm{m}^{-2} \mathrm{~s}^{-1}\right)^{-1}\right]$, respectivamente (figura 4B).

En cuanto a la eficiencia intrínseca del uso del agua (EiUA), se observó una tendencia similar a la EUA, donde los valores más altos se obtuvieron a las $16 \mathrm{~h}$ y $17 \mathrm{~h}$, y los más bajos entre las $11 \mathrm{~h}$ y $13 \mathrm{~h}$, en ambas especies (figura 4C). Los valores oscilaron entre 44,692 [ $(\mu \mathrm{mol}$ $\left.\left.\mathrm{m}^{-2} \mathrm{~s}^{-1}\right)\left(\mathrm{mol} \mathrm{m}^{-2} \mathrm{~s}^{-1}\right)^{-1}\right]$ y $105,917\left[\left(\mu \mathrm{mol} \mathrm{m}^{-2} \mathrm{~s}^{-1}\right)\left(\mathrm{mol} \mathrm{m}^{-2}\right.\right.$ $\left.\left.\mathrm{s}^{-1}\right)^{-1}\right]$ en H. impetiginosus, y entre 39,439 $\left[\left(\mu \mathrm{mol} \mathrm{m}^{-2} \mathrm{~s}^{-1}\right)\right.$ $\left.\left(\mathrm{mol} \mathrm{m}^{-2} \mathrm{~s}^{-1}\right)^{-1}\right]$ y $143,136\left[\left(\mu \mathrm{mol} \mathrm{m} \mathrm{m}^{-2} \mathrm{~s}^{-1}\right)\left(\mathrm{mol} \mathrm{m}^{-2} \mathrm{~s}^{-1}\right)^{-1}\right]$ en H. serratifolius (figura 4C).

La eficiencia instantánea de carboxilación (EiC) mostró un comportamiento similar a la asimilación neta de $\mathrm{CO}_{2}$, aumentando considerablemente a lo largo del día, registrándose valores más altos durante el período de $12 \mathrm{~h}$ y $13 \mathrm{~h}$, con $0,0415\left[\left(\mu \mathrm{mol} \mathrm{m} \mathrm{s}^{-1}\right)\left(\mu \mathrm{mol} \mathrm{de} \mathrm{CO}_{2} \mathrm{~mol}^{-1}\right)^{-1}\right]$ y $0,0376\left[\left(\mu \mathrm{mol} \mathrm{m}^{-2} \mathrm{~s}^{-1}\right)\left(\mu \mathrm{mol} \text { de } \mathrm{CO}_{2} \mathrm{~mol}^{-1}\right)^{-1}\right]$ en H. impe-

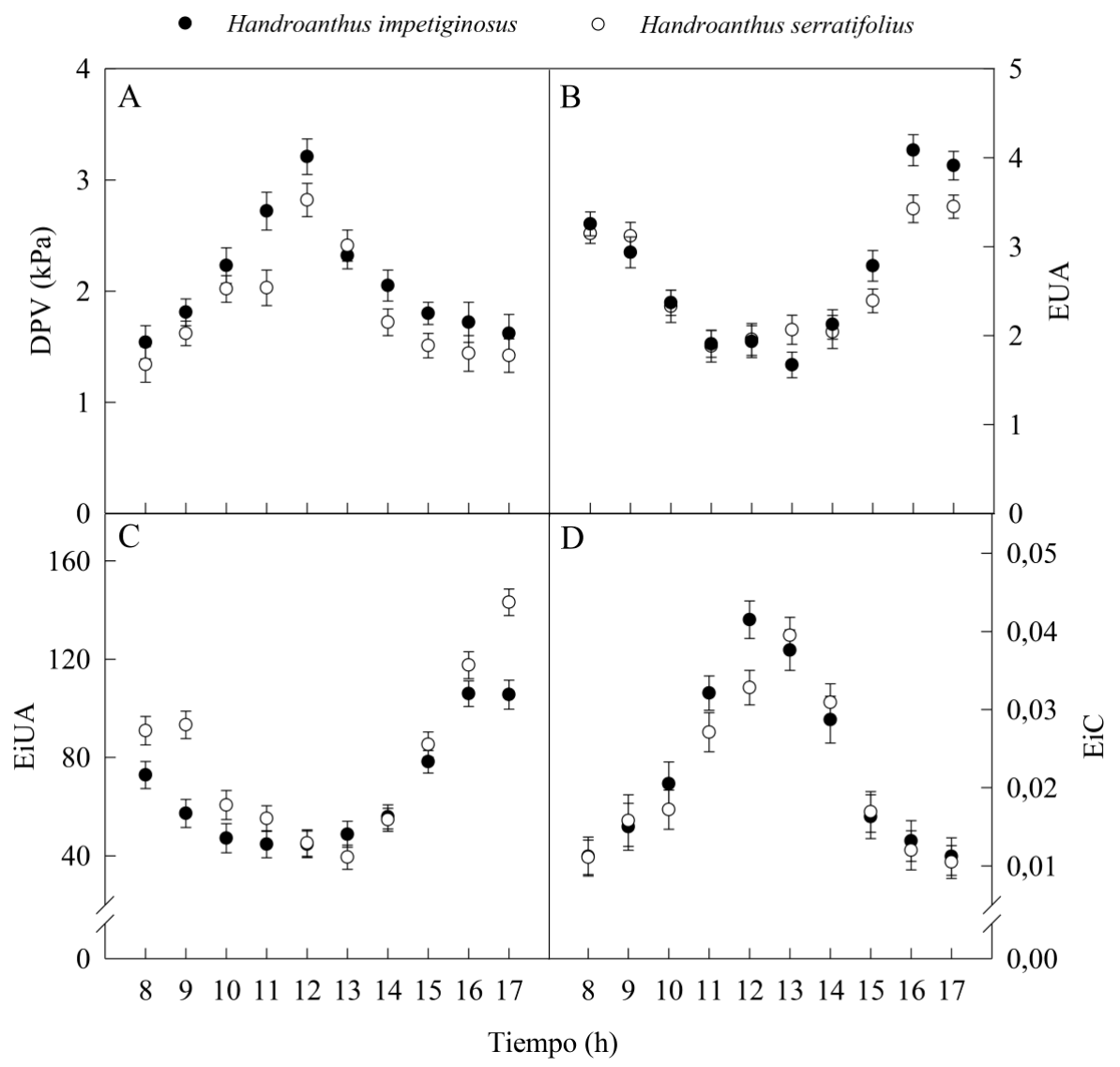

Figura 4. Déficit de presión de vapor (DPV) (A), eficiencia instantánea del uso del agua (EUA) (B), eficiencia intrínseca del uso del agua (EiUA) (C) y eficiencia instantánea de carboxilación (EiC) (D) en plantas de Handroanthus impetiginosus $(\bullet)$ y Handroanthus serratifolius (O) en función de la hora (h) del día.

Vapor pressure deficit (DPV) (A), instantaneous water use efficiency (EUA) (B), intrinsic water use efficiency (EiUA) (C) and instantaneous carboxylation efficiency (EiC) (D) in Handroanthus impetiginosus $(\bullet)$ and Handroanthus serratifolius ( $($ ) plants as a function of time (h) of day. 
tiginosus , y 0,0328 $\left[\left(\mu \mathrm{mol} \mathrm{m} \mathrm{m}^{-2} \mathrm{~s}^{-1}\right)\left(\mu \mathrm{mol} \text { de } \mathrm{CO}_{2} \mathrm{~mol}^{-1}\right)^{-1}\right]$ y $0,0395\left[\left(\mu \mathrm{mol} \mathrm{m} \mathrm{m}^{-2}\right)\left(\mu \mathrm{mol} \text { de } \mathrm{CO}_{2} \mathrm{~mol}^{-1}\right)^{-1}\right]$ en $H$. serratifolius, respectivamente (figura 4D).

\section{DISCUSIÓN}

Los análisis de la prueba de significación Lambda de Wilks y la correlación canónica (ACC) indican que los grupos están asociados de manera dependiente, lo que evidencia el efecto de las variables meteorológicas en los aspectos ecofisiológicos de $H$. impetiginosus y $H$. serratifolius a lo largo del día. En la ACC, la importancia de las variables de cada grupo (meteorológicas y ecofisiológicas) se asocia al mayor coeficiente canónico (cc), es decir, cuanto mayor es el coeficiente, más importante es la variable.

En cuanto a los datos meteorológicos internos y externos del invernadero, Caron et al. (2017) estudiando plantas de Aleurites fordii Hemsl., encontraron resultados similares al presente estudio, con valores máximos de radiación fotosintética activa (RFA) registrados en el período entre 11:30 h y 13:30 h y una elevación de la temperatura del aire entre 13:30 h y 15:30 h. La disminución de la humedad relativa del aire a lo largo del día, posiblemente se produce debido al aumento de la RFA y de la temperatura del aire (Dalmago et al. 2006). La radiación fotosintéticamente activa, combinada con otros factores meteorológicos, puede regular la capacidad fotosintética de las plantas (Taiz et al. 2017).

Entre las 14 y $17 \mathrm{hs}$, en ambas especies disminuyen A y gs, pero aumentó Ci. Este resultado indica que la disminución en la tasa fotosintética no se debió al cierre estomático, ya que evidentemente el $\mathrm{CO}_{2}$ no fue limitante. En ese caso la disminución en A pudo deberse a una menor concentración de ATP y NADPH, debido a una disminución en la etapa fotoquímica de la fotosíntesis (Šigut et al. 2015; Xu et al. 2016; Ribeiro et al. 2020).

El comportamiento de la transpiración (E) para ambas especies fue similar a la tasa de asimilación neta de $\mathrm{CO}_{2}$ y la conductancia estomática, lo que demuestra que esta variable depende directamente de la apertura y cierre de los estomas, así como su asociación a los mecanismos que regulan la temperatura de las hojas y limitación de la pérdida de agua por las hojas (Simões et al. 2015). El aumento de la transpiración en el período de mayor irradiación y temperatura puede haberse producido debido a la mayor eficiencia de las hojas en la asimilación neta del $\mathrm{CO}_{2}$, donde la competencia entre las moléculas de $\mathrm{CO}_{2}$ y $\mathrm{H}_{2} \mathrm{O}$ posiblemente aumentó la pérdida de agua por los estomas (Lambers y Oliveira 2019). La concentración interna de $\mathrm{CO}_{2}$ disminuyó en el período más caluroso del día, posiblemente debido a la alta tasa de fotosíntesis, lo que indica que el $\mathrm{CO}_{2}$ está siendo fijado por Rubisco (Sun et al. 2014). Dalastra et al. (2014), a diferencia del presente estudio, destacaron que las plantas sometidas a condiciones ideales, presentan altos valores de concentración interna de $\mathrm{CO}_{2}$ y asimilación neta de $\mathrm{CO}_{2}$, y que la fotosíntesis se ve limitada por la disminución de la concentración de $\mathrm{CO}_{2}$.

El alto déficit de presión de vapor en el período de mayor RFA y temperatura interna y externa puede estar asociada con una mayor apertura de los estomas, de manera que se produzca un mayor flujo de intercambio de gases en las hojas (Souza et al. 2016). Los valores más altos de EUA y EiUA encontrados a las 16 y $17 \mathrm{~h}$ en ambas especies demuestran que el agua se utilizó de manera más eficiente, lo que indica que hubo una buena absorción de $\mathrm{CO}_{2}$ con una menor pérdida de agua durante este período, además de suponer que el cierre estomático al final de la tarde contribuyó a optimizar el uso del agua por parte de las plantas (Wieser et al. 2018).

La eficiencia instantánea de carboxilación (EiC) se mide por la relación entre la tasa de asimilación neta de $\mathrm{CO}_{2}$ (A) y la concentración interna de $\mathrm{CO}_{2}(\mathrm{Ci})$. Aquí, los valores altos encontrados para $\mathrm{A}$ y $\mathrm{Ci}$ pueden ser indicadores del aumento de la EiC (Silva et al. 2015) producido por la disponibilidad de ATP y NADPH y el sustrato utilizado por Rubisco. Así pues, algunos factores influyen en la EiC, como la cantidad de $\mathrm{CO}_{2}$ que se encuentra en el mesófilo foliar, la temperatura, la luminosidad y la actividad enzimática que permite la plena realización de la fotosíntesis (Silva et al. 2015).

En general, se pudo observar una alta relación entre las variables ecofisiológicas y los factores meteorológicos analizados en ambas especies. Los aspectos ecofisiológicos de las plantas fueron alterados al ser cuantificados en un ambiente protegido (invernadero), en el cual se satisface la demanda de aire evaporativo y limitando evapotranspiración debido a la disminución de la velocidad del viento y la irradiancia a través de la cubierta plástica del ambiente.

El rendimiento fisiológico de $H$. impetiginosus y $H$. serratifolius presentó oscilaciones según las diferentes condiciones meteorológicas a lo largo del día. Por lo tanto, los aspectos ecofisiológicos de ambas especies dependen en gran medida de las condiciones ambientales.

La menor irradiación y la baja temperatura del aire redujeron el comportamiento fisiológico de las plantas $H$. impetiginosus y H. serratifolius.

El momento ideal del día para las evaluaciones de intercambio gaseoso en plantas de $H$. impetiginosus y $H$. serratifolius es entre las 11 a.m. y la 1 p.m.

\section{AGRADECIMIENTOS}

Este estudio fue financiado en parte por la Coordenação de Aperfeiçoamento de Pessoal de Nível Superior - Brasil (CAPES) - Código Financiero 001.

\section{REFERENCIAS}

Alvares CA, JL Stape, PC Sentelhas, JLM Gonçalves, Sparovek G. 2013. Köppen's climate classification map for Brazil. 
Meteorologische Zeitschrift 22: 711-728. DOI: https://doi. org/10.1127/0941-2948/2013/0507

Beltreschi L, RB Lima, DD Cruz. 2019. Traditional botanical knowledge of medicinal plants in a "quilombola" community in the Atlantic Forest of northeastern Brazil. Environment, Development and Sustainability 21: 1185-1203. DOI: https://doi.org/10.1007/s10668-017-0079-6

Benevides DS, FG Carvalho. 2009. Levantamento da flora apícola presente em áreas de caatinga do município de Caraúbas - RN. Sociedade e Território 21: 44-54.

Bhatla SC, MA Lal. 2018. Abiotic Stress. In Bhatla SC, MA Lal eds. Plant Physiology, Development and Metabolism. Singapore, Malaysia. Springer. p. 969-1028.

Caron BO, JR Schneider, EF Elli, E Eloy, VQ Souza. 2017. Physiological relationships in Aleurites fordii Hemsl. seedlings. Revista Árvore 41: 2-7. DOI: https://doi.org/10.1590/1806$\underline{90882017000100002}$

CNCFlora. 2020. Handroanthus in Flora do Brasil $2020 \mathrm{em}$ construção. Jardim Botânico do Rio de Janeiro. Consultado 26 jun. 2020. Disponible en http://floradobrasil.jbrj.gov.br/ reflora/floradobrasil/FB117466

Costa EVS, HPC Brígido, JVS Silva, MR Coelho-Ferreira, GC Brandão, MF Dolabela. 2017. Antileishmanial activity of Handroanthus serratifolius (Vahl) S. Grose (Bignoniaceae). Evidence-Based Complementary and Alternative Medicine 2017: 1-6. DOI: https://doi.org/10.1155/2017/8074275

Cruces E, R Rautenberger, Y Rojas-Lillo, VM Cubillos, N Arancibia-Miranda, E Ramırez-Kushel, I Gomez. 2017. Physiological acclimation of Lessonia spicata to diurnal changing PAR and UV radiation: Differential regulation among down-regulation of photochemistry, ROS scavenging activity and phlorotannins as major photoprotective mechanisms. Photosynthesis Research 131: 145-157. DOI: https://doi.org/10.1007/s11120-016-0304-4

Dalastra GM, MM Echer, VF Guimarães, TL Hachmann, AM Inagaki. 2014. Trocas gasosas e produtividade de três cultivares de meloeiro conduzidas com um e dois frutos por planta. Bragantia 73: 365-371. DOI: https://doi.org/10.1590/1678$\underline{4499.206}$

Dalmago GA, AB Heldwein, AH Nied, EL Grimm, CR Pivetta. 2006. Maximum evapotranspiration of sweet pepper in plastic greenhouse as a function of solar radiation, temperature, relative humidity and water vapor pressure deficit of the air. Ciência Rural 36: 785-792. DOI: https://doi. org/10.1590/S0103-84782006000300010

Dias D, M Pagotto, T Pereira, A Ribeiro. 2017. Estrutura arbórea e sazonalidade da cobertura do dossel em vegetação florestada e aberta no parque nacional serra de Itabaiana, Sergipe, Brasil. Ciência Florestal 27: 719-729. DOI: https://doi. org/10.5902/1980509827757

Ferreira DTRG, EP Gonçalves, JS Viana, LN Ralph, JCA Silva, EM Silva. 2020. Temperature and light under the physiological potential of seeds of Handroanthus impetiginosus. Bioscience Journal 36: 68-77. DOI: https://doi.
org/10.14393/BJ-v36n1a2020-42454

Lambers H, RS Oliveira. 2019. Plant water relations. In Lambers H, RS Oliveira eds. Plant Physiological Ecology. New York, USA. Springer. p. 28-48.

Oliveira AR, CL Boechat, SPN Amorim, MEL Souza, LSL Duarte, HF Silva. 2019. Growth and quality of Handroanthus serratifolius seedlings in soils incorporating amendments and inorganic residues. Ceres 66: 235-242. DOI: $\underline{\mathrm{https}: / /}$ doi.org/10.1590/0034-737x201966030010

Ribeiro JES, AJS Barbosa, SF Lopes, WE Pereira, MB Albuquerque. 2018. Seasonal variation in gas exchange by plants of Erythroxylum simonis Plowman. Acta Botanica Brasílica 32: 287-296. DOI: https://doi.org/10.1590/0102$\underline{33062017 \mathrm{abb} 0240}$

Šigut L, P Holišová, K Klem, M Šprtová, C Calfapietra, MV Marek, V Špunda, O Urban. 2015. Does long-term cultivation of saplings under elevated $\mathrm{CO}_{2}$ concentration influence their photosynthetic response to temperature? Annals of Botany 116: 929-939. DOI: https://doi.org/10.1093/aob/ $\underline{\mathrm{mcv} 043}$

Silva FG, WF Dutra, AF Dutra, IM Oliveira, L Filgueiras, AS Melo. 2015. Gas exchange and chlorophyll fluorescence of eggplant grown under different irrigation depths. Revista Brasileira de Engenharia Agrícola e Ambiental 19: 946952. DOI: https://doi.org/10.1590/1807-1929/agriambi. v19n10p946-952

Simões WL, M Calgaro, DS Coelho, MA Souza, JA Lima. 2015. Respostas de variáveis fisiológicas e tecnológicas da canade-açúcar a diferentes sistemas de irrigação. Revista Ciência Agronómica 46: 11-20.

Souza ER, ACE Amaro, LS Santos, EO Ono, JD Rodrigues. 2016. Fenologia e trocas gasosas da videira cv. Sweet Sunshine em clima semiárido. Comunicata Scientiae 7: 319-333.

Sun Y, L Gu, RE Dickinson, SG Pallardy, J Baker, Y Cao, FM Damatta, X Dong. 2014. Asymmetrical effects of mesophyll conductance on fundamental photosynthetic parameters and their relationships estimated from leaf gas exchange measurements. Plant, Cell \& Environment 37: 978-994. DOI: https://doi.org/10.1111/pce.12213

Taiz L, E Zeiger, IM Møller, A Murphy. 2017. Fisiologia e desenvolvimento vegetal. Porto Alegre, Brazil. Artmed. 858 p.

Vieira C, O Weber. 2017. Saturação por bases de crescimento e nutrição de mudas de Ipê-Amarelo. Floresta e Ambiente 24: 1-10. DOI: $\underline{\text { https://doi.org/10.1590/2179-8087.001916 }}$

Wieser G, W Oberhuber, B Waldboth, A Gruber, R Matyssek, RTW Siegwolf, EE Grams. 2018. Long-term trends in leaf level gas exchange mirror tree-ring derived intrinsic wateruse efficiency of Pinus cembra at treeline during the last century. Agricultural and Forest Meteorology 248: 251258. DOI: https://doi.org/10.1016/j.agrformet.2017.09.023

$\mathrm{Xu}$ Z, Y Jiang, B Jia, G Zhou. 2016. Elevated- $\mathrm{CO}_{2}$ response of stomata and its dependence on environmental factors. Frontiers in Plant Science 7: 1-15. DOI: https://doi.org/10.3389/ fpls.2016.00657 
